\title{
Comprehensiveness-the Need to Resurrect a Sagging Pillar of Primary Care
}

\author{
Tracey L. Henry, MD, MPH, MS ${ }^{\top} \mathbb{D}$, Eugene C. Rich, MD², and Andrew Bazemore, $M D$ \\ $\mathrm{MPH}^{3}$
}

'Division of General Medicine and Geriatrics, Emory University School of Medicine, Faculty Office Bldg, Suite 408, Atlanta, USA; ${ }^{2}$ Center on Health Care Effectiveness, Mathematica, Washington, DC, USA; ${ }^{3}$ Senior Vice President, Research \& Policy, American Board of Family Medicine, Co-Director, Center for Professionalism and Value in Health Care, 1016 16th St NW, Suite 700, Washington, DC, USA.

$J$ Gen Intern Med 37(1):229-31

DOI: $10.1007 / \mathrm{s} 11606-021-07071-2$

(c) Society of General Internal Medicine 2021

\section{INTRODUCTION}

The COVID-19 pandemic highlighted both the importance of primary care and the fragility of its current infrastructure in the United States (US). Within its first 2 months, stark reminders of racial injustice, unaddressed health disparities, and grossly inequitable access to healthcare further underscored the current lack and future importance of universal access to highperforming primary care. At the start of the pandemic, fewer than 1 in 5 Americans could identify a personal usual source of healthcare. ${ }^{1}$ In this time of uncertainty, many patients went without timely care due to a myriad of difficulties. Perhaps chief among these was the lack of an accessible, trusted personal clinician capable of and committed to delivering personalized advice and comprehensive care at a time of unprecedented medical and public health uncertainty. In response to this crisis, changes in actual and virtual visit accessibility to primary care were further complicated by the limited office hours or practice closures imposed by crises in provider organizational finances.

The value of comprehensive primary care has been documented for decades prior to the pandemic. Nonetheless, at its outset, most primary care in the USA occurred in settings adapted to traditional fee-for-service (FFS) payments where physicians provided highly focused face-to-face visits and would often "document and refer" (to specialists) patients with complex conditions. This is in contrast to the type of primary care described by Starfield to have the fundamental features of accessibility, continuity, coordination, and comprehensiveness. $^{2,3}$

\section{Benefits of Comprehensiveness in Primary Care}

While widespread problems with access became immediately evident with the pandemic, comprehensiveness was another

Received April 1, 2021

Accepted July 21, 2021

Published online August 3, 2021 critical feature widely lacking, yet essential to skillful pandemic-era primary care. Comprehensiveness has been core to the definition of primary care since 1967 and is "the feature of primary care ... most salient in distinguishing primary careoriented countries from other countries." 2 Defined as "the extent to which the primary care clinician, as part of the primary care team, recognizes and meets the majority of each patient's physical and common mental health care needs..." prior to the pandemic, the link between comprehensiveness in primary care and outcomes was already well established. ${ }^{2,3}$ Included among the lengthy potential list of comprehensive primary care services are as follows: prevention and wellness, acute care of common health concerns, provision of commonly needed office-based procedures, and appropriate depth and breadth of chronic condition management.

More comprehensive primary care is associated with reduced hospitalizations and emergency department visits, as well as lower costs. ${ }^{4}$ More comprehensive care has also been associated with greater health equity and care continuity. ${ }^{2}$ Since more comprehensive primary care practices meet more patient needs, there are fewer specialty referrals (or selfreferrals) with less health care fragmentation and less need for care coordination across multiple health care providers. ${ }^{2,3}$

\section{Barriers to Comprehensiveness}

While comprehensiveness is deemed essential to high-value primary care, it has not been rewarded under US fee-forservice payment approaches and has declined in recent decades, now varying dramatically across physicians and practices. ${ }^{3,5}$ Despite patients presenting with more complex medical conditions, most primary care has still been delivered through time-constrained visits within outmoded scheduling approaches and reward systems that encourage a piecemeal, non-comprehensive, approach to health care. Even before the pandemic and the recent highlighting of existing health inequities and the importance of unmet social needs on health outcomes, primary care clinicians lacked the time to recognize and address their patients' multiple health needs. Accordingly, various observers have noted that 2020 demonstrated the urgent need to fundamentally reform primary care payment 
to ensure ready access to quality healthcare that includes comprehensive primary care. ${ }^{6}$

In addition to reform of the organization and financing of primary care, primary care clinicians must be trained to deliver comprehensive care. Unfortunately, many primary care clinicians may not be educated in environments conducive to the acquisition of skills for comprehensive primary care. For example, internal medicine training has long been criticized for inconsistent support for primary care, though there are many exemplary primary care internal medicine programs. ${ }^{7}$ Relative to family medicine, internal medicine devotes considerable program time to the care of complex patients including those in hospital settings, but less on the acquisition of the clinical skills needed for comprehensive outpatient primary care. Recent findings suggest this training may be associated with a less comprehensive set of services in internal medicine practices. $^{5}$

The evolution of the health systems in which primary care training occurs may also create educational environments less conducive to comprehensive primary care. The widely reported consolidation of the US health care has resulted in many large hospital systems dominated by specialists delivering lucrative facility-based services. Furthermore, by 2016 , nearly half of all residency positions nationally were controlled by large specialist-dominated hospital systems without a strong link to health profession's education. It is hardly surprising that this environment may encourage referral to specialists over comprehensive primary care, contrary to Starfield's caution that specialty services are more costly than primary care services, both from the systems viewpoint and from the viewpoint of individuals followed over time. ${ }^{2}$

\section{Steps Forward}

With our US population living longer and with more chronic illnesses, it is imperative that the primary care workforce obtains and retains the knowledge and skills to serve the populations for which they are responsible. ${ }^{6}$ Lee et.al suggested that in order to train tomorrow's generalists, we should prioritize and redesign training programs to include teaching comprehensive care management including relevant officebased procedures. ${ }^{8}$ The new NASEM report on Implementing High-Quality Primary Care extended this to "building and supporting interprofessional teams that are accountable for addressing the majority of an individual's health and wellness needs across settings...." Once in practice, these primary care clinicians and teams need the time and the incentives to update their clinical knowledge, as well as the expectations and resources to access needed new clinical information. During COVID-19, such commitment to comprehensive primary care could have mitigated health inequities, maximized access to care for those patients with multiple co-morbidities at highest risk, facilitated timely diagnosis and treatment of health concerns, and promoted continuing care of chronic conditions while reducing unneeded visits and referrals.

\section{Call to Action}

In 2020, COVID-19 further exposed the variability and fragility of comprehensive primary care and the deep inequities in access to it in the USA. The rapid changes in clinical knowledge and challenges to patients obtaining reliable personalized guidance during the pandemic also highlighted why comprehensiveness remains a critical feature of high-quality primary care. Accordingly, the comprehensive primary care practice of the future should be the place where patients receive personalized, skillful, knowledgeable care for the large majority of their health concerns. Ideally, this practice would have the financing and infrastructure to deliver this care even when a new virus alters the diagnostic and treatment landscape and complicates how existing health problems interact with emerging risks. To achieve this goal, clinical training should prepare (and maintenance of certification should support) future primary care clinicians with the diverse competencies needed to thrive in such practices. Educators and clinical practice leaders should endeavor to redesign and reorganize training and practice to restore comprehensiveness to its proper place as a defining characteristic of primary care. Likewise, policymakers must develop and implement payment models that reward more comprehensive primary care and promote reforms in clinician training that ensures skillful provision of comprehensive primary care. Policies ideally should involve all sectors and levels of care to facilitate a community-wide commitment to the delivery of comprehensive, personcentered primary care.

Corresponding Author: Tracey L. Henry, MD, MPH, MS; Division of General Medicine and Geriatrics, Emory University School of Medicine, Faculty Office Bldg, Suite 408, Atlanta, USA (e-mail: Thenry@emory.edu).

\section{Declarations:}

Conflict of Interest: The authors declare that they do not have a conflict of interest.

\section{REFERENCES}

1. Liaw W, Jetty A, Petterson S, Bazemore A, Green L. Trends in the Types of Usual Sources of Care: A Shift from People to Places or Nothing at All. Health Service Res. 2018 Aug;53(4):2346-2367. doi: https://doi.org/10. 1111/1475-6773.12753.

2. Starfield B, Shi L, Macinko J. Contribution of primary care to health systems and health. Milbank Q. 2005;83(3):457-502. doi: https://doi.org/ 10.1111/j.1468-0009.2005.00409.

3. O'Malley AS, Rich EC, Maccarone A, DesRoches CM, Reid RJ. Disentangling the Linkage of Primary Care Features to Patient Outcomes: A Review of Current Literature, Data Sources, and Measurement Needs. Journal of General Internal Medicine. 2015 Suppl 3 (Suppl 3):S576-85. doi: https://doi.org/10.1007/s11606-015-3311-9.

4. Bazemore A, Petterson S, Peterson LE, Phillips RL Jr. More Comprehensive Care Among Family Physicians is Associated with Lower Costs and Fewer Hospitalizations. Annals of Family Medicine. 2015;13(3):206-13. doi: https://doi.org/10.1370/afm.1787.

5. Henry TL, Petterson S, Phillips RS, Phillips RL Jr, Bazemore A. Comparing Comprehensiveness in Primary Care Specialties and Their Effects on Healthcare Costs and Hospitalizations in Medicare Beneficiaries. 
Journal of General Internal Medicine. 2019;34(12):2708-27 10. doi: https:// doi.org/10.1007/s11606-019-05338-3.

6. National Academies of Sciences, Engineering, and Medicine 2021 . Implementing High-Quality Primary Care: Rebuilding the Foundation of Health Care. Washington, DC: The National Academies Press. https://doi. org/ $10.17226 / 25983$.

7. Perkoff GT. Sounding boards. General internal medicine, family practice or something better? N Engl J Med. 1978;299(12):654-7. doi:https://doi. org/10.1056/NEJM197809212991209.
8. Lee PT, Friedberg MW, Bowen JL, Day SC, Kilo CM, Sinsky CA. Training Tomorrow's Comprehensive Primary Care Internists: A Way Forward for Internal Medicine Education. Journal of Graduate Medical Education. 2013;5(2):187-91. doi: https://doi.org/10.4300/JGME-D-12-00134.1.

Publisher's Note: Springer Nature remains neutral with regard to jurisdictional claims in published maps and institutional affiliations. 